\title{
Intestinal Obstruction and Bowel Ischemia due to a Rare Left Paraduodenal Hernia: Case Report
}

\author{
Rani Alsairafi \\ General Surgery Department, College of Medicine, Umm Al-Qura University, Makkah, KSA \\ Email: rasairafi@uqu.edu.sa
}

How to cite this paper: Alsairafi, R. (2022) Intestinal Obstruction and Bowel Ischemia due to a Rare Left Paraduodenal Hernia: Case Report. Surgical Science, 13, 28-33. https://doi.org/10.4236/ss.2022.131005

Received: December 22, 2021

Accepted: January 14, 2022

Published: January 17, 2022

Copyright $\odot 2022$ by author(s) and Scientific Research Publishing Inc. This work is licensed under the Creative Commons Attribution International License (CC BY 4.0).

http://creativecommons.org/licenses/by/4.0/

\begin{abstract}
Bowel obstruction is one of the most common problems found in outpatient departments (OPDs) and emergency rooms. The challenge for surgeons is to be suspicious and recognizing rare disease that could cause bowel obstruction, such as paraduodenal hernia, without delaying the management as the risk of complications increase. This case study presents a patient with bowel obstruction secondary to a paraduodenal hernia.
\end{abstract}

\section{Keywords}

Paraduodenal Hernia, Bowel Obstruction, Bowel Ischemia

\section{Introduction}

An internal hernia is a protrusion of the intra-abdominal organ through a normal or abnormal mesenteric or peritoneal opening. Internal hernias can be either related to congenital peritoneal defects or acquired due to trauma or iatrogenesis.

Internal hernias are rare, and their symptoms are nonspecific, making diagnosis challenging. Computed tomography (CT) scanning is believed to help in the diagnosis of internal hernias and to limit the rate of misdiagnosis [1].

According to Ghahremani's [2] classification, internal abdominal herniations are divided into six groups: paraduodenal hernias (50\% - 55\% of internal abdominal herniations), hernias through the foramen of Winslow (6\% - 10\%), transmesenteric hernias $(8 \%-10 \%)$, pericecal hernias $(10 \%-15 \%)$, intersigmoid hernias $(4 \%-8 \%)$, and paravesical hernias $(<4 \%)$.

A recent systematic review showed that paraduodenal hernias account for $53 \%$ of all internal hernias [3] and cause $0.2 \%-0.9 \%$ of all cases of intestinal ob- 
struction.

This case report is about a left paraduodenal hernia in a 51-year-old male who showed symptoms of bowel obstruction.

\section{Case Report}

A 51-year-old male with an insignificant past medical history entered the emergency department following four days of abdominal pain and vomiting.

The pain was vague, colicky in nature, radiating to the back, and associated with persistent vomiting of mainly gastric juices; there were no alleviating factors, and the pain was not aggravated with food.

On presentation, the patient was in pain. Vitals were within the normal range. Physical examination revealed diffuse abdominal tenderness with slight abdominal distension without any palpable masses; hernial orifices were intact. A digital rectal exam revealed an empty rectum.

Management was initiated at the emergency department with an oxygen supplement, intravenous fluid, a nasogastric tube that drained 1.5 liters on insertion, laboratory workup, and blood gases. An upright chest X-ray and two abdominal view X-rays were ordered. Prophylaxis for a stress ulcer was prescribed.

Laboratory tests showed no noteworthy abnormalities except for mild leukocytosis WBC 13x103, polycythemia Hgb $19 \mathrm{mg} / \mathrm{dl}$, and hematocrit $60 \%$.

An erect chest X-ray showed the air-fluid levels of the small bowel loops and no air under the diaphragm. The computed tomogram of the abdomen showed marked stomach dilatation associated with duodenal and jejunal dilatation with some air fluid level, left mesenteric vessel engorgement, crowding and twisting in the expected herniated sac, and abrupt changes in the caliber of the bowel without any associated mass lesion, significant inflammation, or bowel wall thickening. The scanned ileum and colon appeared normal, with no free air or fluid. The diagnosis was small bowel obstruction secondary to an internal hernia (left paraduodenal) (Figure 1).

The patient was taken to the operating room for emergency laparotomy with COVID-19 precautions. Intraoperative findings were consistent with the paraduodenal internal hernia diagnosis (Figure 2); the hernial sac contained a large segment of small bowel, and the herniated segment was dusky with a $10 \mathrm{~cm}$ ischemic segment that was resected and a blood vessel within the hernial sac that turned out to be the inferior mesenteric artery. Reduction of the hernia and excision of the sac were performed. Second-look surgery was planned, as the viability of one segment was in doubt. The patient was kept intubated and was transferred to the intensive care unit with a planned second-look surgery after 24 hours. In the second-look surgery, both proximal and distal ends were healthy, and side-to-side anastomosis with a linear GIA $60 \mathrm{~mm}$ stapler was done. An abdominal drain was placed. The next day, the patient in the intensive care unit was doing well. Hemodynamically, the patient was stable with no support (pulse $90 \mathrm{~b} / \mathrm{min}$, BP 115/55, mean arterial pressure $70 \mathrm{mmHg}$, afebrile). The patient 


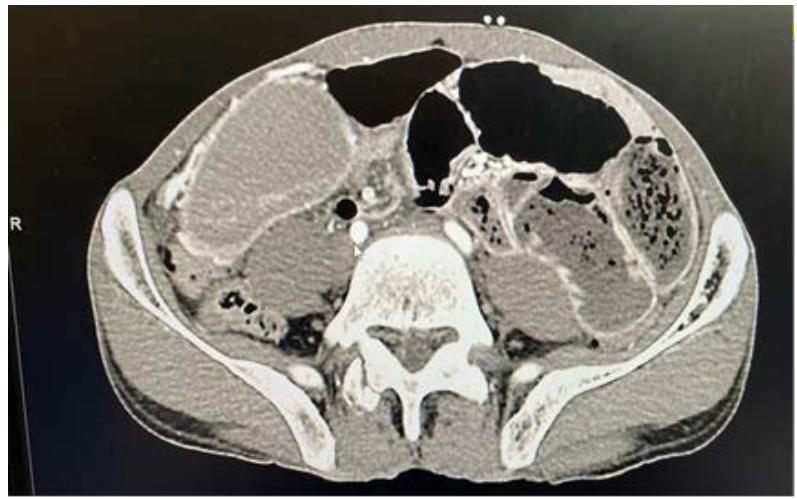

Figure 1. Computed tomogram showing straightening and condensed bowel at the left quadrant of the abdomen.
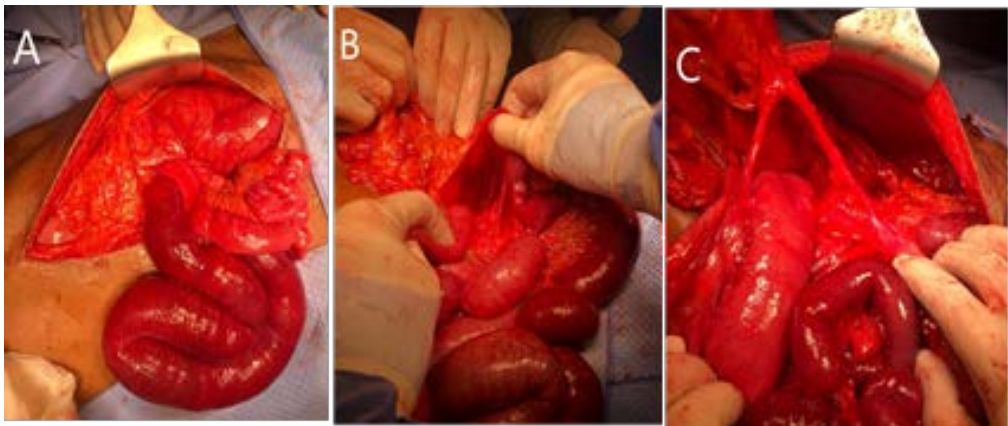

Figure 2. Intraoperative pictures. Picture A shows the sac of paraduodenal hernia with its contents (proximal and distal small bowel). Picture B shows the sac where reduction was done. Picture $\mathrm{C}$ shows the inferior mesenteric artery after dissection from the sac.

was on minimal support on a mechanical ventilator (PEEP 5, $\mathrm{FiO}_{2} 35 \%$, pressure support mode, respiratory rate $18 / \mathrm{min}$ ). Extubated, the patient did well during observation for 24 hours and was transferred to the surgical ward. Postoperative recovery was uneventful. The patient was put on measures to prevent atelectasis, thromboprophylaxis, and stress ulcer prophylaxis and was encouraged to mobilize. On postoperative day three, the patient was stable, was afebrile, had the nasogastric tube removed, and had positive bowel sounds. He consumed $25 \%$ of his daily calorie requirement, taking sips of water, clear fluids, and a bottle of Ensure with each meal. On postoperative day four, the regular diet started with $50 \%$ of his daily calorie requirement and a bottle of Ensure with each meal; the wound was clean.

Histopathology revealed bowel gangrene, with no other pathology detected. The patient was discharged on postoperative day 7 . He was seen in the OPD clinic one week after discharge; nothing of significance was noted and the clips were removed.

\section{Discussion}

Small bowel obstruction is rarely caused by internal hernias, with a reported incidence of $0.2 \%-0.9 \%$. Paraduodenal hernia is rare, with less than 200 cases re- 
ported in the literature, although it is the most common type of internal hernia that causes intestinal obstruction [3] [4]. Paraduodenal hernia occurs due to a congenital defect during embryonic development of the midgut, with the prearterial portion failing to rotate normally and becoming trapped behind the colonic mesentery, creating a peritoneal pocket that contains the small bowel and the distal ileum [5].

The incidence is higher in males than in females. Most patients with paraduodenal hernias develop abdominal pain, dyspepsia, and distention. When intra-abdominal pressure increases, the small intestine may enter the duodenal recess. The jejunum is the most common herniating viscus. The contents of the hernia are usually the small intestine or stomach and rarely the colon [6].

The mortality rate associated with intestinal strangulation in patients with symptomatic left paraduodenal hernia ranges from $20 \%$ to $50 \%$ in the published literature; an accurate preoperative diagnosis of paraduodenal hernia is necessary because of the potentially fatal complications [7]. Contrast-enhanced abdominal CT imaging, which has an accuracy of $90 \%-95 \%$ and a sensitivity of $94 \%-100 \%$, plays an essential role in the preoperative diagnosis of internal hernias, which show capsulated loops of small bowel and air fluid level secondary to entrapment, anterior and left upward displacement of the superior mesenteric vein, and abnormal take-off of the superior or inferior mesenteric artery [8] [9]. Angiography can also be used in cases of left paraduodenal hernia to identify displaced jejunal arteries superiorly and to the left. Barium meal can also provide a definitive diagnosis of paraduodenal hernia. Preoperative serial filming is essential to diagnosis because it can show that the inferior mesenteric vein and ascending left colic artery lie in the anteromedial border of the left paraduodenal hernia, findings best appreciated during arteriography or laparotomy [8] [9] [10] [11].

The optimal treatment of a paraduodenal hernia is surgical correction, due to the risk of incarceration in untreated cases (50\%) [12]. Surgical correction of a paraduodenal hernia is mainly done by reducing the small bowel and closing the hernial opening [5]. The laparoscopic approach has advantages over the open approach, thanks to less postoperative pain, early mobilization, smaller scars, shorter hospital stays, and reduced postoperative morbidity [13]. This patient was managed by an emergent open surgical approach with a good postoperative recovery that was free of complications. He was discharged from the hospital after seven days, with no complications at the routine outpatient follow-up.

\section{Conclusion}

This case report examined a left paraduodenal hernia in a 51-year-old male with delayed presentation. The hernia was diagnosed by abdominal CT, and the patient was operated on with a good clinical outcome. Although internal hernias are rare, late presentation or delayed diagnosis may lead to bowel perforation and peritonitis, which increase the risk of mortality. The possibility of a para- 
duodenal hernia should be considered with the absence of any history of intra-abdominal inflammatory disease, external hernia, or surgery, whenever an intestinal obstruction is suspected. CT imaging increases the chance of early diagnosis. After the text edit has been completed, the paper is ready for the template.

\section{Acknowledgements}

I hereby would like to thank the surgical team worked with me and provided high standard of care during the hospitalization of the patient:

Dr. Alanood Shisha, Dr. Khalid Al Qurshi, Dr. Abdulelah Almalki, Dr. Waleed Tashkandi, Dr. Ahmad Alsalmi, Dr. Mansour Sheban, Dr. Fahad AlKhyzae, and Dr. Samaher Khedadi.

\section{Conflicts of Interest}

The author declares no conflicts of interest regarding the publication of this paper.

\section{References}

[1] Mathieu, D., Luciani, A. and GERMAD Group (2004) Internal Abdominal Herniations. American Journal of Roentgenology, 183, 397-404. https://doi.org/10.2214/ajr.183.2.1830397

[2] Ghahremani, G.G. (1984) Internal Abdominal Hernias. Surgical Clinics of North America, 64, 393-406. https://doi.org/10.1016/S0039-6109(16)43293-7

[3] Schizas, D., Apostolou, K., Krivan, S., et al. (2019) Paraduodenal Hernias: A Systematic Review of the Literature. Hernia, 23, 1187-1197. https://doi.org/10.1007/s10029-019-01947-3

[4] Blachar, A., Federle, M.P. and Dodson, S.F. (2001) Internal Hernia: Clinical and Imaging Findings in 17 Patients with Emphasis on CT Criteria. Radiology, 218, 68-74. https://doi.org/10.1148/radiology.218.1.r01ja5368

[5] Bartlett, M.K., Wang, C. and Williams, W.H. (1968) The Surgical Management of Paraduodenal Hernia. Annals of Surgery, 168, 249-254. https://doi.org/10.1097/00000658-196808000-00010

[6] Liu, T.H. (2016) Timing of Abdominal CT Evaluation Impacts the Diagnosis of Paraduodenal Hernia. The American Surgeon, 82, 546-549. https://doi.org/10.1177/000313481608200617

[7] Dritsas, E.R., Ruiz, O.R., Kennedy, G.M., Blackford, J. and Hasl, D. (2001) Paraduodenal Hernia: A Report of Two Cases. The American Surgeon, 67, 733.

[8] Dayananda, L., Sreekumar, K.P., Moorthy, S. and Prabhu, N.K. (2006) Para Duodenal Hernias: A Pictorial Essay. Indian Journal of Radiology and Imaging, 16, 469-471. https://doi.org/10.4103/0971-3026.32248

[9] Selçuk, D., Kantarci, F., Oğüt, G. and Korman, U. (2005) Radiological Evaluation of Internal Abdominal Hernias. The Turkish Journal of Gastroenterology: The Official Journal of Turkish Society of Gastroenterology, 16, 57-64.

[10] Khan, M.A., Lo, A.Y. and Vande Maele, D.M. (1998) Paraduodenal Hernia. The American Surgeon, 64, 1218-1222.

[11] Kuzinkovas, V., Haghighi, K., Singhal, R. and Andrews, N.J. (2008) Paraduodenal 
Hernia: A Rare Cause of Abdominal Pain. Canadian Journal of Surgery, 51, E127.

[12] Muneer, H., Jawad, A. and Al Aradi, J. (2017) Left Paraduodenal Hernia. Bahrain Medical Bulletin, 39, 250-252. https://doi.org/10.12816/0047781

[13] Doishita, S., Takeshita, T., Uchima, Y., et al. (2016) Internal Hernias in the Era of Multidetector CT: Correlation of Imaging and Surgical Findings. Radiographics, 36, 88-106. https://doi.org/10.1148/rg.2016150113 